\title{
PASCHKE'S CONJECTURE FOR THE ENDPOINT ANISOTROPIC SERIES REPRESENTATIONS OF THE FREE GROUP
}

\author{
M. GABRIELLA KUHN and TIM STEGER
}

(Received 12 December 2000; revised 23 November 2001)

Communicated by G. Willis

\begin{abstract}
Let $\Gamma$ be a free noncommutative group with free generating set $A_{+}$. Let $\mu \in \ell^{\prime}(\Gamma)$ be real, symmetric, nonnegative and suppose that $\operatorname{supp}(\mu)=A_{+} \cup A_{+}^{-1}$. Let $\lambda$ be an endpoint of the spectrum of $\mu$ considered as a convolver on $\ell^{2}(\Gamma)$. Then $\lambda-\mu$ is in the left kernel of exactly one pure state of the reduced $C_{\mathrm{reg}}^{*}(\Gamma)$; in particular, Paschke's conjecture holds for $\lambda-\mu$.
\end{abstract}

2000 Mathematics subject classification: primary 20E05, $22 \mathrm{D} 25$.

Keywords and phrases: free group, irreducible unitary representation, pure state.

\section{Introduction}

Let $\Gamma$ be a noncommutative free group on finitely many generators. Choose a basis for $\Gamma$ and denote by $|\cdot|$ the length with respect to this basis. Let $A$ consist of the basis elements and of their inverses. Each $x \in \Gamma$ can be uniquely represented as a reduced word, that is, a product $a_{1} a_{2} \cdots a_{n}$ of elements of $A$ with $a_{j} a_{j+1} \neq e$. Let $q+1=|A|$.

The reduced $C^{*}$-algebra of $\Gamma$ is the $C^{*}$-algebra generated by the left regular representation $\pi_{\text {reg }}$, that is, the completion of the convolution algebra of finitely supported functions with respect to the norm

$$
\|f\|_{C_{\mathrm{rg}}^{*}(\Gamma)}^{2}=\left\|\pi_{\mathrm{reg}}(f)\right\|^{2}=\sup _{\|g\|_{\ell^{2}(\Gamma)}=1}\langle f * g, f * g\rangle .
$$

A unitary representation $\pi$ of $\Gamma$ is weakly contained in $\pi_{\text {reg }}$ if for every finitely supported function $f$ on $\Gamma$ one has $\|\pi(f)\| \leq\left\|\pi_{\mathrm{reg}}(f)\right\|$, where $\|\cdot\|$ denotes the

This research was supported by the Italian CNR.

(C) 2003 Australian Mathematical Society 1446-7887/03 $\$ A 2.00+0.00$ 
operator norm of $\pi(f)$; in other words $\pi$ is weakly contained in $\pi_{\text {reg }}$ if and only if $\pi$ extends to a representation of $C_{\mathrm{reg}}^{*}(\Gamma)$. In our case, using Power's result [8] that $C_{\text {reg }}^{*}(\Gamma)$ is simple, the above condition is equivalent to saying $\|\pi(f)\|=\left\|\pi_{\text {reg }}(f)\right\|$. From this point on every representation is assumed to be unitary and weakly contained in $\pi_{\text {reg. }}$.

Fix a nonzero, finitely supported function $\mu^{\prime} \in \ell^{2}(\Gamma)$. In [6] Paschke conjectures that $\mu^{\prime}$ lies in the left kernel of at most finitely many pure states of $C_{\mathrm{reg}}^{*}(\Gamma)$. In terms of representations this means that

(a) there are only finitely many equivalence classes of irreducible representations $\pi$, weakly contained in $\pi_{\text {reg }}$, such that $\pi\left(\mu^{\prime}\right)$ has nontrivial kernel, and

(b) for any such $\pi, \operatorname{dim} \operatorname{ker} \pi\left(\mu^{\prime}\right)=1$.

It is convenient for our exposition to fix $\mu \in \ell^{2}(\Gamma)$ and let $\mu^{\prime}=\lambda-\mu=\lambda \delta_{e}-\mu$ for $\lambda \in \mathbb{C}$.

Let $A_{+} \subseteq A$ be a set of free generators of $\Gamma$. Let $n=\sharp\left(A_{+}\right)=(q+1) / 2$. Paschke treats the case $\mu=\mathbf{1}_{A_{+}}=\sum_{a \in A_{+}} \delta_{a}$. For each $\lambda, 0 \leq \lambda \leq \sqrt{n}$, he constructs an irreducible representation $\pi_{\lambda}$ so that $\operatorname{dim} \operatorname{ker} \pi_{\lambda}(\lambda-\mu)=1$. No such representation can occur for $\lambda>\sqrt{n}$ because $\sqrt{n}$ is the spectral radius of $\mu$. In the case $\lambda=\sqrt{n}$, but only in that case, he proves that $\pi_{\lambda}$ is, up to equivalence, the unique irreducible representation such that $\pi(\lambda-\mu)$ has nontrivial kernel. Thus, for $\lambda=\sqrt{n}$, the conjecture is proved. In [7] Paschke extends his results to the analogous anisotropic situation.

In this paper we shall prove that the conjecture is true in the case

$$
\mu=\sum_{a \in A} p_{a} \delta_{a}, \quad \lambda=\mu_{0}
$$

where $p_{a}(a \in A)$ are positive numbers with $p_{a}=p_{a^{-1}}, \sum_{a \in A} p_{a}=1$ and $\mu_{0}$ is the right endpoint of the $\ell^{2}(\Gamma)$ spectrum of $\mu$. The case of the left endpoint, $-\mu_{0}$, reduces trivially to the case of $+\mu_{0}$ by taking tensor products with the character $\chi_{0}$ such that $\chi_{0}(a)=-1$ for each generator $a \in A$. For analogous reasons, Paschke's results hold not only for $\lambda=\sqrt{n}$, but also for $\lambda=\sqrt{n} e^{i \theta}$.

In fact we shall prove a stronger result, like Paschke's - there is, up to equivalence, only one irreducible representation for which $\pi(\mu) v=\mu_{0} v$ for some nonzero vector $v$, namely the one in the anisotropic principal series of Figà-Talamanca and Steger [2]. The case $p_{a}=1 /(q+1)$ for all $a \in A$ corresponds to the isotropic principal series of Figà-Talamanca and Picardello [1].

In the present case, as in Paschke's case, the conjecture is proved only for the extreme value of $\lambda$. Referring to our paper [5] for the definition of perfect boundary realization, observe that Paschke's representation $\pi_{\lambda}$ for $\lambda=\sqrt{n}$ admits exactly one boundary realization, which is perfect. The same holds for the anisotropic principal series representation corresponding to the endpoint, $\lambda=\mu_{0}$. In neither case does this 
emain true as one passes to the interior of the spectrum. In spite of these notable joints in common, our techniques are quite different from Paschke's. Ours definitely ton't apply to his case, and it is not clear how his might apply to our case.

The techniques developed here are based on the growth of the matrix coefficients of $\tau$ and give a positive answer to the question every time that matrix coefficients grow as fast as they can. This happens for the endpoint representations of the isotropic or anisotropic principal series. A central role is played by Haagerup's inequality [3].

THEOREM 1.1. Let $(\pi, H)$ be a unitary representation of $\Gamma$. Suppose that $v \in H$ is cyclic for $\pi$ and consider the positive definite function $\phi(x)=\langle\pi(x) v, v\rangle$. The following conditions are equivalent

(a) $\pi$ is weakly contained in the regular representation.

(b) $\sum_{|x|=n}|\phi(x)|^{2} \leq(n+1)^{2}\|v\|^{4}$.

(c) For every positive $\epsilon$ the function $x \rightarrow \phi(x) e^{-\epsilon|x|}$ belongs to $\ell^{2}(\Gamma)$.

Observe first that, by polarization, every matrix coefficient $\rho(x)=\left\langle\pi(x) v, v^{\prime}\right\rangle$ can be written as a finite linear combination of positive definite functions so that if we replace $\phi(x)$ with $\left\langle\pi(x) v, v^{\prime}\right\rangle$ (c) remains true while (with the same arguments used in [3]) (b) becomes

$$
\sum_{|x|=n}\left|\left\langle\pi(x) v, v^{\prime}\right\rangle\right|^{2} \leq C(n+1)^{2}\|v\|^{2}\left\|v^{\prime}\right\|^{2} .
$$

Let $\left(\pi_{1}, H_{1}\right),\left(\pi_{2}, H_{2}\right)$ be two irreducible representations of $\Gamma$ weakly contained in the regular representation. Fix vectors $v$ in $H_{1}$ and $w$ in $H_{2}$ with $\|v\|=\|w\|=1$. Define a sesquilinear form on $H_{2} \times H_{1}$ by letting

$$
B_{\epsilon}\left(w^{\prime}, v^{\prime}\right)=\sum_{x \in \Gamma}\left(\pi_{1}(x) v, v^{\prime}\right\rangle \overline{\left\langle\pi_{2}(x) w, w^{\prime}\right\rangle} e^{-\epsilon|x|} .
$$

By the Cauchy-Schwartz inequality

$$
\begin{aligned}
& \sum_{|x|=n}\left|\left\langle\pi_{1}(x) v, v^{\prime}\right\rangle \overline{\left\langle\pi_{2}(x) w, w^{\prime}\right\rangle}\right| e^{-\epsilon|x|} \\
& \quad \leq\left(\sum_{|x|=n}\left|\left\langle\pi_{1}(x) v, v^{\prime}\right\rangle\right|^{2} e^{-\epsilon|x|}\right)^{1 / 2}\left(\sum_{|x|=n}\left|\left\langle\pi_{2}(x) w, w^{\prime}\right\rangle\right|^{2} e^{-\epsilon|x|}\right)^{1 / 2} \\
& \quad \leq C(n+1)^{2} e^{-\epsilon n}\left\|v^{\prime}\right\|\left\|w^{\prime}\right\| .
\end{aligned}
$$

Thus $B_{\epsilon}$ is well defined.

Moreover, since $\sum_{n=0}^{\infty}(n+1)^{2} x^{n}=(1+x) /(1-x)^{3}$ for $|x|<1$ we have

$$
\left|B_{\epsilon}\left(w^{\prime}, v^{\prime}\right)\right| \leq C \sum_{n=0}^{\infty}(n+1)^{2} e^{-\epsilon n}\left\|v^{\prime}\right\|\left\|w^{\prime}\right\|=C\left(1+e^{-\epsilon}\right) \frac{\left\|v^{\prime}\right\|\left\|w^{\prime}\right\|}{\left(1-e^{-\epsilon}\right)^{3}} .
$$


Hence $\epsilon^{3} B_{\epsilon}$ is bounded as $\epsilon$ goes to 0 . See Corollary 3.2 below for a proof that if

$$
\underset{\epsilon \rightarrow 0}{\limsup } \epsilon^{3}\left|B_{\epsilon}\left(w^{\prime}, v^{\prime}\right)\right|>0
$$

then the two representations are equivalent and moreover a version of Schur orthogonality holds for limits of normalized sums of products of matrix coefficients.

The problem is that in many known cases the limit in (1) is zero. For example, using the most natural choices for the vectors, $B_{\epsilon}$ grows like $1 / \epsilon$ in the case of a nonendpoint isotropic ([1]) or anisotropic ([2]) spherical series representation. Growth like $1 / \epsilon^{2}$ is to be found for Paschke's endpoint representation $(\lambda=\sqrt{n})$ and for the representations of our 1996 paper [4].

There are a few known cases in which the growth of the quantity $B_{\epsilon}\left(v^{\prime}, w^{\prime}\right)$ is of the magnitude of $1 / \epsilon^{3}$, leading to a nonzero limit for $\epsilon^{3} B_{\epsilon}$. These cases are

- when $\pi=\pi_{z_{0}}$ is an endpoint representation of the isotropic spherical series (corresponding to the value $z_{0}=1 / 2+i k \pi$ in the notation of [1])

- when $\pi=\pi_{ \pm \mu_{0}}$ is an endpoint representation of the anisotropic principal series of [2].

In these cases the spherical functions $\langle\pi(x) v, v\rangle$ grow as fast as Haagerup's results allow them to and we can get precise and interesting information about the eigenspace of the operator $\pi\left(\mu-\lambda_{0}\right)$.

\section{The spherical functions}

Fix once and for all a free set $A_{+}$of generators and let $A=A_{+} \cup A_{-}$consist of the generators and of their inverses. The Cayley graph of $\Gamma$ with respect to $A$ is a homogeneous tree of degree $q+1=|A|$. Each vertex is labelled with a group element and the (unoriented) edges are given by the pairs $\{x, x a\}$ where $a \in A$.

The anisotropic series representations were defined by Figà-Talamanca and Steger in the memoir [2] for all discrete groups whose Cayley graph is a tree. Those groups are free product of $M$ copies of $\mathbb{Z}$ and $N$ copies of $\mathbb{Z}_{2}$, where $2 M+N=q+1$. In the paper mentioned above, the case $N=q+1$ was considered in detail. Nonetheless, formulas and results hold unchanged if we pass from a free product of $q+1$ copies of $\mathbb{Z}_{2}$ to a free group on $(q+1) / 2$ generators.

Choose positive numbers $p_{a}(a \in A)$ with $\sum_{a \in A} p_{a}=1$ and $p_{a}=p_{a^{-1}}$. Let $\mu=\sum_{a \in A} p_{a} \delta_{a}$. Consider the operator of right convolution by $\mu$ acting on $\ell^{2}(\Gamma)$. Its spectrum is a real interval $\left[-\mu_{0}, \mu_{0}\right]$ and, for $\lambda \notin\left[-\mu_{0}, \mu_{0}\right]$, the resolvent is given by right convolution with the Green function $g_{\lambda}(x)=(\lambda-\mu)^{-1}(x)$ which is positive for $\lambda$ real and greater than $\mu_{0}$. 
Let $x=a_{1} a_{2} \cdots a_{n}$ be the reduced word expression for $x$. Set

Then

$$
g_{\lambda}(e)=\frac{1}{2 w(\lambda)} \text {. }
$$

$$
g_{\lambda}(x)=\frac{1}{2 w(\lambda)} \xi_{a_{1}}(\lambda) \xi_{a_{2}}(\lambda) \cdots \xi_{a_{n}}(\lambda)
$$

where $\left\{\xi_{a_{j}}(\lambda)\right\}_{j=1}^{q+1}$ and $w(\lambda)$ are algebraic functions of $\lambda$ which are positive for $\lambda$ real ind greater than $\mu_{0}$ and where $\xi_{a_{j}}=\xi_{a_{j}^{-1}}$.

It is more convenient to turn $w(\lambda)$ and $\xi_{a_{j}}(\lambda)$ into functions of $w$ : the formulas selow can be found in [2, page 10] and are valid for large positive values of $w$.

$$
\begin{aligned}
\lambda & =-(q-1) w+\sum_{a \in A} \sqrt{w^{2}+p_{a}} \\
\xi_{a} & =\left(\sqrt{w^{2}+p_{a}}-w\right) / p_{a}
\end{aligned}
$$

There is a unique positive $w_{0}$ for which we have $d \lambda /\left.d w\right|_{w_{0}}=0$. The point $\lambda\left(w_{0}\right)=\mu_{0}$ is the (right) endpoint of the spectrum of $\mu$ and it is also a branch point for the analytic function $w(\lambda)$ (see the discussion in [2, pages 22-27]).

At this point we also have $d^{2} \lambda /\left.d w^{2}\right|_{w_{0}}=L>0$ so that

$$
\lambda=\mu_{0}+\frac{L}{.2}\left(w-w_{0}\right)^{2}+\cdots .
$$

Set $\sqrt{\lambda-\mu_{0}}=\epsilon$ and use $\epsilon$ as a parameter for the formulae in [2]. In a neighbour$\operatorname{rood}$ of $\lambda=\mu_{0}$ we have

3) $w$ is an analytic function of $\epsilon, w\left(\mu_{0}\right)>0, d w /\left.d \epsilon\right|_{\mu_{0}}=l>0$,

4) $\xi_{a}$ is an analytic function of $\epsilon, \xi_{a}\left(\mu_{0}\right)>0, d \xi_{a} /\left.d \epsilon\right|_{\mu_{0}}=-k_{a}<0$,

$$
\begin{aligned}
g_{\lambda}^{2}(e) & =(\lambda-\mu)^{-2}(e)=\frac{-d(\lambda-\mu)^{-1}}{d \lambda}(e)=\frac{-d}{d \lambda} \frac{1}{2 w(\lambda)} \\
& =\frac{l}{2\left(w\left(\mu_{0}\right)\right)^{2}} \frac{1}{2 \epsilon}+O(1) .
\end{aligned}
$$

Let $\pi_{\lambda}$ be the representation of the anisotropic principal series for $\mu$ which corresponds to some $\lambda \in\left[-\mu_{0}, \mu_{0}\right]$. Note that $\pi_{\lambda}$ is irreducible. There is a special vector 1 $n$ the representation space of $\pi_{\lambda}$. Actually, 1 is the function identically 1 in the realzation of $\pi_{\lambda}$ acting on $L^{2}(\Omega, d \nu)$ given in [2]. One has $\pi_{\lambda}(\mu) 1=\lambda 1$. The spherical inctions are first defined as usual by $\phi_{\lambda}(x)=\left\langle\pi_{\lambda}(x) \mathbf{1}, \mathbf{1}\right\rangle$ for $\lambda \in\left(-\mu_{0}, \mu_{0}\right)$. When $\imath=\mu_{0}$ the spherical function can be computed as a limit, see [2, page 30]

$$
\phi_{\mu_{0},}(x)=\lim _{\lambda \rightarrow \mu_{0}}\left\langle\pi_{\lambda}(x) \mathbf{1}, \mathbf{1}\right\rangle=\frac{\left.\frac{d}{d \epsilon}\right|_{\lambda=\mu_{0}}(\lambda-\mu)^{-1}(x)}{\left.\frac{d}{d \epsilon}\right|_{\lambda=\mu_{0}}(\lambda-\mu)^{-1}(e)}
$$

ind analogously for $\lambda=-\mu_{0}$. 


\section{The results}

In order to prove our results we need to modify a little bit the factor $e^{-\epsilon|x|}$ which guarantees the convergence of $\sum_{x \in \Gamma}\left\langle\pi_{1}(x) v, v^{\prime}\right\rangle \overline{\left\langle\pi_{2}(x) w, w^{\prime}\right\rangle} e^{-\epsilon|x|}$. In the case of the isotropic principal series, no modification is necessary.

LEMMA 3.1. Let $\psi_{\epsilon}(x)$ be any family of complex functions having the properties:

(a) $\left|\psi_{\epsilon}(x)\right| \leq C e^{-c \epsilon|x|}$ for some positive constants $C$ and $c$.

(b) $\left|\psi_{\epsilon}(x)-\psi_{\epsilon}(x a)\right| \leq K(\epsilon) e^{-c \epsilon|x|}$ for each $a \in A$ where $K(\epsilon) \rightarrow 0$ as $\epsilon \rightarrow 0^{+}$.

Assume that $\left(\pi_{1}, H_{1}\right)$ and $\left(\pi_{2}, H_{2}\right)$ are representations weakly contained in the regular representation. Suppose that for some $v_{1}, w_{1} \in H_{1}$ and $v_{2}, w_{2} \in H_{2}$ we have that

$$
\underset{\epsilon \rightarrow 0}{\limsup } \epsilon^{3}\left|\sum_{x \in \Gamma}\left\langle\pi_{1}(x) v_{1}, w_{1}\right\rangle \overline{\left\langle\pi_{2}(x) v_{2}, w_{2}\right\rangle} \psi_{\epsilon}(x)\right|>0 .
$$

Then there is a $\Gamma$-intertwiner $J: H_{2} \rightarrow H_{1}$ so that $\left\langle J w_{2}, w_{1}\right\rangle \neq 0$.

PROOF. We prove first that $\lim \sup _{\epsilon \rightarrow 0^{+}} \epsilon^{3}\left|\sum_{x \in \Gamma}\left\langle\pi_{1}(x) v_{1}, v_{1}^{\prime}\right\rangle \overline{\left\langle\pi_{2}(x) v_{2}, v_{2}^{\prime}\right\rangle} \psi_{\epsilon}(x)\right|$ is finite and that we can form the analogue of $B_{\epsilon}\left(w^{\prime}, v^{\prime}\right)$ by replacing $e^{-\epsilon|x|}$ with $\psi_{\epsilon}(x)$. In fact condition (a) together with Haagerup's inequality says that for any $\pi_{1}$ and $\pi_{2}$ weakly contained in $\pi_{\text {reg }}$ we have

$$
\begin{aligned}
& \sum_{|x|=n}\left|\left\langle\pi_{1}(x) v_{1}, v_{1}^{\prime}\right\rangle \overline{\left\langle\pi_{2}(x) v_{2}, v_{2}^{\prime}\right\rangle} \psi_{\epsilon}(x)\right| \\
& \quad \leq\left(\sum_{|x|=n}\left|\left\langle\pi_{1}(x) v_{1}, v_{1}^{\prime}\right\rangle\right|^{2}\left|\psi_{\epsilon}(x)\right|\right)^{1 / 2}\left(\sum_{|x|=n}\left|\left\langle\pi_{2}(x) v_{2}, v_{2}^{\prime}\right\rangle\right|^{2}\left|\psi_{\epsilon}(x)\right|\right)^{1 / 2} \\
& \quad \leq C(n+1)^{2} e^{-c \epsilon n}\left\|v_{1}^{\prime}\right\|\left\|v_{2}^{\prime}\right\|\left\|v_{1}\right\|\left\|v_{2}\right\| .
\end{aligned}
$$

Adding up over $n$ we get

$$
\left|\sum_{x \in \Gamma}\left\langle\pi_{1}(x) v_{1}, v_{1}^{\prime}\right\rangle \overline{\left\langle\pi_{2}(x) v_{2}, v_{2}^{\prime}\right\rangle} \psi_{\epsilon}(x)\right| \leq 2 C \frac{\left\|v_{1}^{\prime}\right\|\left\|v_{2}^{\prime}\right\|\left\|v_{1}\right\|\left\|v_{2}\right\|}{\left(1-e^{-c \epsilon}\right)^{3}} .
$$

Consider $v_{1}$ and $v_{2}$ from (6) to be fixed. For every $\epsilon>0$, define $J_{\epsilon}: H_{2} \rightarrow H_{1}$ by letting $\left\langle J_{\epsilon} v_{2}^{\prime}, v_{1}^{\prime}\right\rangle=\epsilon^{3} \sum_{x \in \Gamma}\left\langle\pi_{1}(x) v_{1}, v_{1}^{\prime}\right\rangle \overline{\left\langle\pi_{2}(x) v_{2}, v_{2}^{\prime}\right\rangle} \psi_{\epsilon}(x)$.

The inequality (7) bounds the operator norm of $J_{\epsilon}$ as $\epsilon \rightarrow 0$. Choose a sequence $\left\{\epsilon_{j}\right\}$ tending to zero such that $\left\langle J_{\epsilon_{j}} w_{2}, w_{1}\right\rangle$ has a nonzero limit, then extract a weak operator convergent subsequence of $\left\{J_{\epsilon_{j}}\right\}$ with limit $J$. This is possible since norm closed balls are compact in the weak operator topology and $H_{i}$ are separable. 
For some suitable sequence $\left\{\epsilon_{j}\right\}$, we have

$$
\left\langle J\left(v_{2}^{\prime}\right), v_{1}^{\prime}\right\rangle=\lim _{j} \epsilon_{j}^{3} \sum_{x \in \Gamma}\left\langle\pi_{1}(x) v_{1}, v_{1}^{\prime}\right\rangle \overline{\left\langle\pi_{2}(x) v_{2}, v_{2}^{\prime}\right\rangle} \psi_{\epsilon_{j}}(x) .
$$

By a construction $\left\langle J w_{2}, w_{1}\right\rangle \neq 0$. Now we show that $J$ intertwines $\pi_{2}$ to $\pi_{1}$. It is enough to check that $J \pi_{2}(a)=\pi_{1}(a) J$ when $a$ is a generator of $\Gamma$. Let us compute $\left\langle J\left(\pi_{2}(a) v_{2}^{\prime}\right), v_{1}^{\prime}\right\rangle-\left\langle\pi_{1}(a) J\left(v_{2}^{\prime}\right), v_{1}^{\prime}\right\rangle:$

$$
\begin{aligned}
& \left|\left\langle J \pi_{2}(a)\left(v_{2}^{\prime}\right), v_{1}^{\prime}\right\rangle-\left\langle\pi_{1}(a) J\left(v_{2}^{\prime}\right), v_{1}^{\prime}\right\rangle\right| \\
& =\left|\left\langle J \pi_{2}(a)\left(v_{2}^{\prime}\right), v_{1}^{\prime}\right\rangle-\left\langle J\left(v_{2}^{\prime}\right), \pi_{1}\left(a^{-1}\right) v_{1}^{\prime}\right\rangle\right| \\
& =\lim _{j} \epsilon_{j}^{3} \mid \sum_{x \in \Gamma}\left(\pi_{1}(x) v_{1}, v_{1}^{\prime}\right\rangle \overline{\left\langle\pi_{2}(x) v_{2}, \pi_{2}(a) v_{2}^{\prime}\right\rangle} \psi_{\epsilon_{j}}(x) \\
& -\sum_{x \in \Gamma}\left\langle\pi_{1}(x) v_{1}, \pi_{1}\left(a^{-1}\right) v_{1}^{\prime}\right\rangle \overline{\left\langle\pi_{2}(x) v_{2}, v_{2}^{\prime}\right\rangle} \psi_{\epsilon_{j}}(x) \mid \\
& =\lim _{j} \epsilon_{j}^{3} \mid \sum_{x \in \Gamma}\left\langle\pi_{1}(a x) v_{1}, v_{1}^{\prime}\right\rangle \overline{\left\langle\pi_{2}(x) v_{2}, v_{2}^{\prime}\right\rangle} \psi_{\epsilon_{j}}(a x) \\
& -\sum_{x \in \Gamma}\left\langle\pi_{1}(a x) v_{1}, v_{1}^{\prime}\right\rangle \overline{\left\langle\pi_{2}(x) v_{2}, v_{2}^{\prime}\right\rangle} \psi_{\epsilon_{j}}(x) \mid \\
& \leq \limsup _{j} \epsilon_{j}{ }^{3} K\left(\epsilon_{j}\right) \sum_{x \in \Gamma}\left|\left\langle\pi_{1}(x) v_{1}, \pi_{1}\left(a^{-1}\right) v_{1}^{\prime}\right\rangle \overline{\left\langle\pi_{2}(x) v_{2}, v_{2}^{\prime}\right\rangle}\right| e^{-\epsilon \epsilon_{j}|x|}=0 .
\end{aligned}
$$

COROLLARY 3.2. Let $\psi_{\epsilon}$ be a family of functions satisfying conditions (a) and (b) of Lemma 3.1. Let $(\pi, H)$ be an irreducible representation. Then there exist a sequence $\epsilon_{j} \rightarrow 0$ and a constant $C_{0} \geq 0$ so that for any $v_{1}, v_{1}^{\prime}, v_{2}, v_{2}^{\prime} \in H$

$$
\lim _{j} \epsilon_{j}^{3} \sum_{x \in \Gamma}\left\langle\pi_{1}(x) v_{1}, v_{1}^{\prime}\right\rangle \overline{\left\langle\pi_{2}(x) v_{2}, v_{2}^{\prime}\right\rangle} \psi_{\epsilon_{j}}(x)=C_{0}\left\langle v_{1}, v_{2}\right\rangle \overline{\left\langle v_{1}^{\prime}, v_{2}^{\prime}\right\rangle}
$$

PROOF. This is a corollary to the demonstration rather than to the statement of Lemma 3.1. If the lim sup of (6) is zero for all choices of the vectors, then Corollary 3.2 is true with $C_{0}=0$. Otherwise, we fix some $v_{1}$ and $v_{2}$ and construct $J: H \rightarrow H$ as in the proof of Lemma 3.1. Since $\pi$ is irreducible, $J$ is necessarily scalar, say $J=C_{0}^{\prime}=C_{0}^{\prime}\left(v_{1}, v_{2}\right)$. Then for all $v_{1}^{\prime}$ and $v_{2}^{\prime}$ we have

$$
C_{0}^{\prime}\left(v_{1}, v_{2}\right) \overline{\left\langle v_{1}^{\prime}, v_{2}^{\prime}\right\rangle}=\left\langle J v_{2}^{\prime}, v_{1}^{\prime}\right\rangle=\lim _{j} \epsilon_{j}^{3} \sum_{x \in \Gamma}\left\langle\pi_{1}(x) v_{1}, v_{1}^{\prime}\right\rangle \overline{\left\langle\pi_{2}(x) v_{2}, v_{2}^{\prime}\right\rangle} \psi_{\epsilon_{j}}(x) .
$$

The rest of the proof proceeds as for the usual Schur orthogonality relations. Exchanging the roles of $\left(v_{1}, v_{2}\right)$ and $\left(v_{1}^{\prime}, v_{2}^{\prime}\right)$ we find that $C_{0}^{\prime}$ is proportional to $\left\langle v_{1}, v_{2}\right\rangle$, 
say $C_{0}^{\prime}\left(v_{1}, v_{2}\right)=\left\langle v_{1}, v_{2}\right\rangle C_{0}$. Then for all $v_{1}, v_{2}, v_{1}^{\prime}, v_{2}^{\prime} \in H$

$$
C_{0}\left\langle v_{1}, v_{2}\right\rangle \overline{\left\langle v_{1}^{\prime}, v_{2}^{\prime}\right\rangle}=\lim _{j} \epsilon_{j}^{3} \sum_{x \in \Gamma}\left\langle\pi_{1}(x) v_{1}, v_{1}^{\prime}\right\rangle \overline{\left\langle\pi_{2}(x) v_{2}, v_{2}^{\prime}\right\rangle} \psi_{\epsilon_{j}}(x) .
$$

Taking $v_{1}=v_{1}^{\prime}, v_{2}=v_{2}^{\prime}$ we find that $C_{0}>0$.

THEOREM 3.3. Suppose that $(\pi, H)$ is an irreducible representation weakly contained in $\pi_{\text {reg. }}$. Assume that there exists a nonzero vector $v \in H$ such that $\pi(\mu) v=\mu_{0}$. Then $\pi$ is equivalent to the representation $\left(\pi_{\mu_{0}}, H_{\mu_{0}}\right.$ ) corresponding to the endpoint of the anisotropic principal series of [2]. Moreover dim ker $\pi_{\mu_{0}}\left(\mu_{0}-\mu\right)=1$.

ProOF. Fix any nonzero eigenvector $v$ of $\pi(\mu)$ corresponding to the eigenvalue $\mu_{0}$. Let 1 be the nonzero $\mu_{0}$ eigenvector of $\pi_{\mu_{0}}(\mu)$ as described previously. We shall construct a function $\psi_{\epsilon}$ satisfying (a) and (b) of Lemma 3.1 for which

$$
\lim _{\epsilon \rightarrow 0} \epsilon^{3} \sum_{x \in \Gamma}\left\langle\pi_{\mu_{0}}(x) \mathbf{1}, \mathbf{1}\right\rangle \overline{\langle\pi(x) v, v)} \psi_{\epsilon}(x) \neq 0 .
$$

Let $g_{\lambda}(x)=(\lambda-\mu)^{-1}(x)$ be the resolvent of $\mu$. Choose $\lambda$ positive and greater than $\mu_{0}$ and set $\epsilon=\sqrt{\lambda-\mu_{0}}$. Define

$$
\psi_{\epsilon}(x)=\frac{(\lambda-\mu)^{-2}(x)}{(\lambda-\mu)^{-2}(e)\left\langle\pi_{\mu_{0}}(x) 1,1\right\rangle}=\frac{g_{\lambda}^{2}(x)}{g_{\lambda}^{2}(e)} \frac{1}{\phi_{\mu_{0}}(x)} .
$$

Since $\pi(\mu) v=\mu_{0} v$ the functional calculus gives $\pi\left((\lambda-\mu)^{-2}\right) v=v /\left(\lambda-\mu_{0}\right)^{2}$. One computes

$$
\begin{aligned}
\sum_{x \in \Gamma}\left\langle\pi_{\mu_{0}}(x) \mathbf{1}, \mathbf{1}\right\rangle \overline{\langle\pi(x) v, v\rangle} \psi_{\epsilon}(x) & =\sum_{x \in \Gamma}\langle\pi(x) v, v\rangle \frac{(\lambda-\mu)^{-2}(x)}{(\lambda-\mu)^{-2}(e)} \\
& =\frac{1}{g_{\lambda}^{2}(e)}\left\langle\pi\left((\lambda-\mu)^{-2}\right) v, v\right\rangle=\frac{\langle v, v\rangle}{g_{\lambda}^{2}(e)\left(\lambda-\mu_{0}\right)^{2}} .
\end{aligned}
$$

So,

$$
\lim _{\epsilon} \epsilon^{3} \sum_{x \in \Gamma}\left\langle\pi_{\mu_{0}}(x) 1,1\right\rangle \overline{\langle\pi(x) v, v\rangle} \psi_{\epsilon}(x)=\lim _{\lambda \rightarrow \mu_{0}} \frac{\left(\sqrt{\lambda-\mu_{0}}\right)^{3}}{g_{\lambda}^{2}(e)\left(\lambda-\mu_{0}\right)^{2}}\langle v, v\rangle
$$

By (5) the quantity $(\lambda-\mu)^{-2}(e)=g_{\lambda}^{2}(e)$ behaves like $l /\left(4 \sqrt{\lambda-\mu_{0}} w^{2}\left(\mu_{0}\right)\right)$ so that the above limit is

$$
\frac{4 w^{2}\left(\mu_{0}\right)}{l}\langle v, v\rangle \neq 0
$$


Taking for granted that $\psi_{\epsilon}$ satisfies conditions (a) and (b) of Lemma 3.1, said lemma guarantees the existence of a $\Gamma$-intertwiner $J: H_{\mu_{0}} \rightarrow H$ such that $\langle J v, \mathbf{1}\rangle \neq 0$. As $\pi$ and $\pi_{\mu_{0}}$ are irreducible, $\pi$ is unitarily equivalent to $\pi_{\mu_{0}}$.

Suppose that $\operatorname{dim} \operatorname{ker} \pi_{\mu_{0}}\left(\mu_{0}-\mu\right)>1$. In the above argument, choose $\pi=\pi_{\mu_{0}}$ and choose $v \in \operatorname{ker} \pi_{\mu_{0}}\left(\mu_{0}-\mu\right)$ so that $\langle v, \mathbf{1}\rangle=0$. Then $J$ intertwines $H_{\mu_{0}}$ to itself so that $\langle J v, \mathbf{1}\rangle \neq 0$. As $J$ is necessarily a scalar, this is a contradiction. (Alternatively, [2] contains a direct, computational proof that $\operatorname{dim} \operatorname{ker} \pi_{\mu_{0}}\left(\mu_{0}-\mu\right)=1$.)

Now we prove that $\psi_{\epsilon}$ satisfies (a) and (b) of Lemma 3.1. First note that for $\lambda>\mu_{0}$

(8) $\frac{(\lambda-\mu)^{-2}(x)}{(\lambda-\mu)^{-2}(e)}=\frac{d\left((\lambda-\mu)^{-1}(x)\right) / d \lambda}{d\left((\lambda-\mu)^{-1}(e)\right) / d \lambda}=\frac{d\left((\lambda-\mu)^{-1}(x)\right) / d \epsilon}{d\left((\lambda-\mu)^{-1}(e)\right) / d \epsilon}=\frac{d g_{\lambda}(x) / d \epsilon}{d g_{\lambda}(e) / d \epsilon}$,

where $\epsilon=\sqrt{\lambda-\mu_{0}}$. Fix $x=a_{1} \cdots a_{n}$, an element of length $n$ in $\Gamma$. Define $\xi(x)$, a function of $\lambda$, by $\xi(x)=\xi_{a_{1}}(\lambda) \ldots \xi_{a_{n}}(\lambda)$. Using (8) and (2) we compute

$$
\frac{(\lambda-\mu)^{-2}(x)}{(\lambda-\mu)^{-2}(e)}=\xi(x)\left(1+\frac{w}{d w / d \epsilon} \sum_{j=1}^{n} \frac{-d \xi_{a_{j}} / d \epsilon}{\xi_{a_{j}}}\right) .
$$

Since $\phi_{\mu_{0}}(x)$ is given by the right hand side of (8) evaluated at $\lambda=\mu_{0}$,

$$
\psi_{\epsilon}(x)=\frac{(\lambda-\mu)^{-2}(x)}{(\lambda-\mu)^{-2}(e) \phi_{\mu_{0}}(x)}=\frac{\left.\xi(x)\right|_{\lambda}}{\left.\xi(x)\right|_{\mu_{0}}}\left(\frac{1+\left.\left.\frac{w}{d w / d \epsilon}\right|_{\lambda} \sum_{j=1}^{n} \frac{-d \xi_{a_{j}} / d \epsilon}{\xi_{a_{j}}}\right|_{\lambda}}{1+\left.\left.\frac{w}{d w / d \epsilon}\right|_{\mu_{0}} \sum_{j=1}^{n} \frac{-d \xi \xi_{a_{j}} / d \epsilon}{\xi_{a_{j}}}\right|_{\mu_{0}}}\right) .
$$

Using (3) and (4) one sees that for $\lambda$ in a neighbourhood of $\mu_{0}$ the functions $w$, $d w / d \epsilon, \xi_{a}$, and $-d \xi_{a} / d \epsilon$ are positive and differentiable with respect to $\epsilon$. It follows that there exist constants $h, \epsilon_{0}>0$ so that

$$
1-h \epsilon \leq \frac{w(\lambda)}{w\left(\mu_{0}\right)} \cdot \frac{d w /\left.d \epsilon\right|_{\mu_{0}}}{d w /\left.d \epsilon\right|_{\lambda}} \cdot \frac{\xi_{a}\left(\mu_{0}\right)}{\xi_{a}(\lambda)} \cdot \frac{-d \xi_{a} /\left.d \epsilon\right|_{\lambda}}{-d \xi_{a} /\left.d \epsilon\right|_{\mu_{0}}} \leq 1+h \epsilon
$$

for $0<\epsilon \leq \epsilon_{0}$ and for all $a \in A$. Hence

$$
1-h \epsilon \leq \frac{1+\left.\left.\frac{w}{d w / d \epsilon}\right|_{\lambda} \sum_{j=1}^{n} \frac{-d \xi_{a_{j}} / d \epsilon}{\xi_{a_{j}}}\right|_{\lambda}}{1+\left.\left.\frac{w}{d w / d \epsilon}\right|_{\mu_{0}} \sum_{j=1}^{n} \frac{-d \xi_{a_{j}} / d \epsilon}{\xi_{a_{j}}}\right|_{\mu_{0}}} \leq 1+h \epsilon
$$

According to (4) $d \xi_{a} /\left.d \epsilon\right|_{\mu_{0}}=-k_{a}<0$. Hence there exist constants $H, c>0$ so that

$$
1-H \epsilon \leq \frac{\xi_{a}(\lambda)}{\xi_{a}\left(\mu_{0}\right)} \leq e^{-\epsilon \epsilon}
$$


for all $0<\epsilon \leq \epsilon_{0}$ and for all $a \in A$. Consequently,

$$
\left(\left.\xi(x)\right|_{\lambda}\right) /\left(\left.\xi(x)\right|_{\mu_{0}}\right) \leq e^{-c \epsilon|x|} .
$$

Together with (9) and (10), this proves condition (a) in Lemma 3.1.

Now we prove condition (b). Fix $a \in A$ so that $|a x|=n+1$. The opposite case, $|a x|=n-1$, easily reduces to this one. From (9) we obtain

$$
\begin{aligned}
& \psi_{\epsilon}(a x)-\psi_{\epsilon}(x)
\end{aligned}
$$

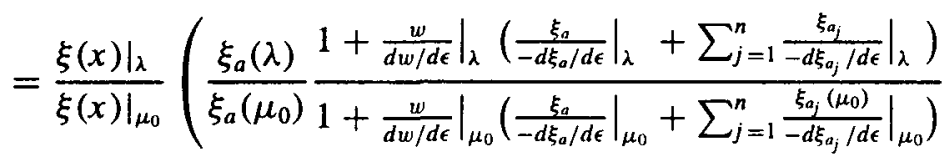

$$
\begin{aligned}
& \left.-\frac{1+\left.\frac{w}{d w / d \epsilon}\right|_{\lambda}\left(\left.\sum_{j=1}^{n} \frac{\xi_{\sigma_{j}}}{-d \xi_{a_{j}} / d \epsilon}\right|_{\lambda}\right)}{1+\left.\frac{w}{d w / d \epsilon}\right|_{\mu_{0}}\left(\left.\sum_{j=1}^{n} \frac{\xi_{a_{j}}}{-d \xi_{a_{j}} / d \epsilon}\right|_{\mu_{0}}\right)}\right) .
\end{aligned}
$$

By (10) and (11)

$$
\begin{aligned}
((1-H \epsilon)(1-h \epsilon)-(1+h \epsilon)) \frac{\left.\xi(x)\right|_{\lambda}}{\left.\xi(x)\right|_{\mu_{0}}} & \leq \psi_{\epsilon}(a x)-\psi_{\epsilon}(x) \\
& \leq((1+h \epsilon)-(1-h \epsilon)) \frac{\left.\xi(x)\right|_{\lambda}}{\left.\xi(x)\right|_{\mu_{0}}}
\end{aligned}
$$

which means that

$$
-(2 h+H) \epsilon \frac{\left.\xi(x)\right|_{\lambda}}{\left.\xi(x)\right|_{\mu_{0}}} \leq \psi_{\epsilon}(a x)-\psi_{\epsilon}(x) \leq 2 h \epsilon \frac{\left.\xi(x)\right|_{\lambda}}{\left.\xi(x)\right|_{\mu_{0}}} .
$$

Together with (12), this completes the proof of condition (b).

\section{References}

[1] A. Figà-Talamanca and A. M. Picardello, Harmonic analysis on free groups, Lecture Notes in Pure and Appl. Math. 87 (Marcel Dekker, New York, 1983).

[2] A. Figà-Talamanca and T. Steger, 'Harmonic analysis for anisotropic random walks on homogeneous trees', Mem. Amer. Math. Soc. 110 (1994), No. 531.

[3] U. Haagerup, 'An example of a nonnuclear $C$ "-algebra which has the metric approximation property', Invent. Math. 50 (1979), 279-293.

[4] M. G. Kuhn and T. Steger, 'More irreducible boundary representations of free groups', Duke Math. J. 82 (1996), $381-436$.

[5] _ 'Monotony of certain free group representations', J. Funct. Anal. 179 (2001), 1-17.

[6] W. Paschke, 'Pure eigenstates for the sum of generators of the free group', Pacific J. Math. 197 (2001), 151-171. 
[7] — 'Some irreducible free group representations in which a linear combination of the generators has an eigenvalue', J. Aust. Math. Soc. 72 (2002), 257-286.

[8] R. T. Powers, 'Simplicity of the $C^{*}$-algebra associated with the free group on two generators', Duke Math. J. 42 (1975), 151-156.

Dipartimento di Matematica Università di Milano "Bicocca"

Viale Sarca 202

20126 Milano

Italia

e-mail: kuhn@matapp.unimib.it
Struttura di Matematica e Fisica

Università di Sassari

Via Vienna 2

07100 Sassari

Italia

e-mail: steger@ssmain.uniss.it 
